\title{
The maximum principle for minimal varieties of arbitrary codimension
}

\author{
BRIAN WHITE
}

\begin{abstract}
We prove that an $m$-dimensional minimal variety in a Riemannian manifold cannot touch the boundary at a point where the sum of the smallest $m$ principal curvatures is greater than 0 . We prove a stronger maximum principle in case the variety is a hypersurface. We also prove analogous results for varieties with bounded mean curvature.
\end{abstract}

Let $N$ be a smooth Riemannian manifold with boundary. In general, $N$ need not be complete. Suppose $X$ is a compactly supported $C^{1}$ tangent vectorfield on $N$ such that

$$
X \cdot \nu_{N} \geq 0
$$

at all points of $\partial N$, where $\nu_{N}$ is the unit normal to $\partial N$ that points into $N$. Then $X$ generates a one-parameter family $t \in[0, \infty) \mapsto \phi_{t}$ of maps of $N$ into itself such that $\phi_{0}$ is the identity map and such that

$$
\frac{d}{d t} \phi_{t}(\cdot)=X\left(\phi_{t}(\cdot)\right)
$$

If $V$ is a $C^{1}$ submanifold of $N$ with finite area, we let $\delta V(X)$ denote the first variation of area of $V$ with respect to $X$ :

$$
\delta V(X)=\left(\frac{d}{d t}\right)_{(t=0)} \operatorname{area}\left(\phi_{t}(V)\right) .
$$

More generally, if $V$ has locally finite area, we let

$$
\delta V(X)=\left(\frac{d}{d t}\right)_{(t=0)} \operatorname{area}\left(\phi_{t}(V \cap W)\right),
$$

where $W$ is any open subset of $N$ that contains the support of $X$ and that has compact closure. Even more generally, $V$ can be any varifold in $N$. (The theorems in this paper are stated for arbitrary varifolds, but readers 
unfamiliar with varifolds may subsititute " $C^{1}$ submanifold" for "varifold" throughout the paper. The appendix to [8] is a brief introduction to varifolds that contains most of what is needed here. For a more thorough treatment, see [6] or [1].)

We say that a varifold $V$ in $N$ minimizes area to first order in $N$ provided

$$
\delta V(X) \geq 0
$$

for every compactly supported $C^{1}$ tangent vectorfield $X$ on $N$ satisfying (1). In particular, any smooth minimal submanifold of $N$ or, more generally, any stationary varifold in $N$ minimizes the area to first order in $N$.

We say that $N$ is strongly $m$-convex at a point $p \in \partial N$ provided

$$
\kappa_{1}+\kappa_{2}+\cdots+\kappa_{m}>0
$$

where $\kappa_{1} \leq \kappa_{2} \leq \cdots \leq \kappa_{n-1}$ are the principal curvatures of $\partial N$ at $p$ with respect to the unit normal $\nu_{N}$ that points into $N$. We say that $N$ is $m$-convex at $p$ provided $\kappa_{1}+\kappa_{2}+\cdots+\kappa_{m} \geq 0$.

Theorem 1 (Maximum Principle for Minimal Varifolds). Let $N$ be a smooth Riemannian manifold with boundary, and let $p$ be a point in $\partial N$ at which $N$ is strongly $m$-convex. Then $p$ is not contained in the support of any $m$-dimensional varifold in $N$ that minimizes area to first order in $N$. Indeed, there is an $\epsilon>0$ such that

$$
\operatorname{dist}(p, \operatorname{spt} V) \geq \epsilon
$$

for all such varifolds $V$.

In case $V$ is a smooth minimal submanifold, the fact that $V$ cannot contain $p$ was proved by Jorge and Tomi [4]. Indeed, they proved that if $N$ is $m$-convex (not necessarily strongly $m$-convex) at all boundary points, then a smooth, connected minimal $m$-dimensional submanifold cannot touch $\partial N$ unless it lies entirely in $\partial N$. An analogous result for smooth submanifolds $V$ of bounded mean curvature was proved by Dierkes and Schwab [2] assuming (in addition to the appropriate condition on $\partial N$ ) that $N$ is flat. The proofs here are similar to the proofs in those papers. The results here are stronger in that they apply to nonsmooth varieties (varifolds), and in that the varieties are not assumed to be stationary with respect to all variations, but only with respect to variations that take $N$ into itself (i.e., that satisfy (1)). 
This extra generality is important because when one solves variational problems for area in a manifold-with-boundary $N$, the solution surfaces need not be stationary (or minimal), but rather only stationary with respect to variations satisfying (1). Likewise, even if a solution surface turns out to be a differentiable manifold, it need not be smooth — it may only be $C^{1,1}$. Of course if the boundary of $N$ is strictly $m$-convex, then according to Theorem 1, the solution surface does lie in the interior of $N$, which implies that it is stationary with respect to all variations, and that if it is a differentiable manifold, then it is a smooth submanifold. But it is important that these properties of the surface are conclusions of Theorem 1 rather than hypotheses.

Theorem 1 is proved by constructing a suitable test vectorfield $X$.

Theorem 2. Let $N$ be a smooth Riemannian manifold with boundary. Let $p$ be a point in $\partial N$ and let $\eta<\kappa_{1}+\cdots+\kappa_{m}$, where $\kappa_{1} \leq \cdots \leq \kappa_{n-1}$ are the principal curvatures of $\partial N$ at $p$ with respect to the unit normal $\nu_{N}$ that points into $N$. Then there is a compactly supported $C^{\infty}$ vectorfield $X$ on $N$ such that $X(p)$ is a nonzero normal to $\partial N$, such that

$$
X \cdot \nu_{N} \geq 0 \text { at all points of } \partial N
$$

and such that

$$
\delta V(X) \leq-\eta \int|X| d \mu_{V}
$$

for every $m$-dimensional varifold $V$ in $N$.

Here $\mu_{V}$ is the weight measure associated with $V$. (If $V$ is a $C^{1}$ submanifold, then the integration in (3) is simply integration over $V$ with respect to $m$-dimensional area.)

We remark that $X$ can be chosen so that its support is contained in an arbitrarily small neighborhood of $p$ and so that the vectorfield $X /|X|$ (wherever $X$ is nonzero) is arbitrarily $C^{0}$-close to $\nabla \operatorname{dist}(\cdot, \partial N)$. (In the proof below, one simply chooses $\epsilon$ sufficiently small.)

To see that Theorem 1 follows from Theorem 2, note that if $N$ is strongly $m$-convex at $p$, then we may choose the $\eta$ in Theorem 2 to be positive. If $V$ minimizes area to first order in $N$, then by definition and by Theorem 2 ,

$$
0 \leq \delta V(X) \leq-\eta \int|X| d \mu_{V}
$$


Since $\eta>0$, this implies that $|X|$ vanishes $\mu_{V}$ almost everywhere and thus that the support of $V$ cannot contain any point where $X \neq 0$. Hence $\operatorname{dist}(p, \operatorname{spt} V) \geq \epsilon$, where $\epsilon$ is the distance from $p$ to the nearest point where $X$ vanishes.

Proof of Theorem 2. Given a compactly supported $C^{1}$ vectorfield $X$ on $N$, let $\Psi_{X}: N \rightarrow \mathbf{R}$ be the function

$$
\Psi_{X}(x)=\max (\operatorname{trace}(\nabla X \mid P)),
$$

where the maximum is over all $m$-dimensional linear subspaces $P$ of $\operatorname{Tan}_{x} N$ and where

$$
\operatorname{trace}(\nabla X \mid P)=\sum_{i=1}^{m} \mathbf{u}_{i} \cdot \nabla_{\mathbf{u}_{i}} X
$$

for any orthonormal basis $\mathbf{u}_{1}, \ldots, \mathbf{u}_{m}$ of $P$.

If $V$ is an $m$-dimensional $C^{1}$ submanifold of $N$, then by the first variation formula $[6, \S 9.3]^{1}$

$$
\begin{aligned}
\delta V(X) & =\int \operatorname{trace}\left(\nabla X \mid \operatorname{Tan}_{x} V\right) d \mu_{V} x \\
& \leq \int \Psi_{X} d \mu_{V} .
\end{aligned}
$$

More generally, if $V$ is any $m$-dimensional varifold in $N$, then by the first variation formula $[6, \S 39.2]$,

$$
\begin{aligned}
\delta V(X) & =\int_{(x, P) \in G_{m}(N)} \operatorname{trace}(\nabla X \mid P) d V(x, P) \\
& \leq \int_{(x, P) \in G_{m}(N)} \Psi_{X}(x) d V(x, P) \\
& =\int \Psi_{X} d \mu_{V},
\end{aligned}
$$

where $G_{m}(N)$ is the set of pairs $(x, P)$ such that $x \in N$ and $P$ is an $m$-dimensional linear subspace of $\operatorname{Tan}_{x} N$.

\footnotetext{
${ }^{1}$ Equation (4) is proved by expressing the area of $\phi_{t}(V)$ as the integral of a Jacobian determinant and then differentiating under the integral sign. If $V$ is a smooth submanifold, one can then integrate by parts to express $\delta V(X)$ as $-\int X \cdot H d \mu_{V}$.
} 
Thus, we see that the conclusion (3) of Theorem 2 will hold provided

$$
\Psi_{X}(\cdot) \leq-\eta|X(\cdot)|
$$

at all points of $N$.

For constructing the desired vectorfield $X$, we may assume that $N$ is part of a larger Riemannian manifold $\tilde{N}$ (without boundary) of the same dimension. ${ }^{2}$ Let

$$
\Sigma=\left\{q \in \tilde{N}: \operatorname{dist}(x, N)=\operatorname{dist}(x, p)^{4}\right\}
$$

Note that $\Sigma$ and $\partial N$ make second-order contact at $p$. By replacing $\tilde{N}$ with a small geodesic ball around $p$, we may assume that $\Sigma$ is a smooth hypersurface and that there is smooth, well-defined nearest-point retraction from $\tilde{N}$ to $\Sigma$. (We will later replace $\tilde{N}$ by an even smaller ball to ensure that additional conditions are satisfied.)

For $x \in \tilde{N}$, let $u(x)$ be the signed distance from $x$ to $\Sigma$, with the sign chosen so that $u$ is nonnegative on $N$. For $q \in \tilde{N}$, let $\Sigma_{q}$ be the level set of $u$ that contains $q$. Note that $\nu(q):=\nabla u(q)$ is a unit normal to $\Sigma_{q}$. Let

$$
k_{1}(q) \leq \cdots \leq k_{n-1}(q)
$$

be the principal curvatures of $\Sigma_{q}$ at $q$ with respect to the unit normal $\nu(q)$.

Note that

$$
k_{1}+\cdots+k_{m}>\eta
$$

at $p$ since $\Sigma$ and $\partial N$ make second-order contact at $p$. By replacing $\tilde{N}$ with a sufficiently small ball around $p$, we may assume that (7) holds at all points of $\tilde{N}$, that

$$
\nu \cdot \nu_{N}>0 \text { at all points of } \partial N
$$

and that the $\left|k_{i}\right|$ are uniformly bounded:

$$
\left|k_{i}(q)\right| \leq K \quad(q \in \tilde{N}, i \leq n-1)
$$

\footnotetext{
${ }^{2}$ If the existence of a such an $\tilde{N}$ is not clear, note that $p$ has a neighborhood diffeomorphic to a closed half-space in $\mathbf{R}^{n}$. Since Theorem 2 is local, we can assume that $N$ is that half-space with some smooth Riemannian metric. We can extend the Riemannian metric to all of $\mathbf{R}^{n}$ and then let $\tilde{N}$ be $\mathbf{R}^{n}$ with the extended metric.
} 
Let $\epsilon$ be a positive number (to be specified later), and define a vectorfield $X$ on $N$ by

$$
X(\cdot)=\phi(u(\cdot)) \nu(\cdot)
$$

where

$$
\phi(t)= \begin{cases}\exp \left(\frac{1}{t-\epsilon}\right) & \text { if } 0 \leq t<\epsilon \\ 0 & \text { if } t \geq \epsilon\end{cases}
$$

(We need not define $\phi(t)$ for $t<0$ since $u \geq 0$ on $N$.) Note that

$$
\frac{\phi^{\prime}(t)}{\phi(t)}=\frac{-1}{(t-\epsilon)^{2}} \leq \frac{-1}{\epsilon^{2}}
$$

for $0 \leq t<\epsilon$, and thus

$$
\phi^{\prime}(t) \leq-\frac{1}{\epsilon^{2}} \phi(t)
$$

for all $t \geq 0$. Thus by choosing $\epsilon \leq K^{-1 / 2}$, we can ensure that

$$
\phi^{\prime}(t) \leq-K \phi(t)
$$

for all $t \geq 0$.

We also choose $\epsilon$ small enough such that $N \cap\{u \leq \epsilon\}$ is compact.

We claim that the vectorfield $X$ has the desired properties. First note that

$$
\operatorname{spt} X=\overline{N \cap\{u<\epsilon\}}
$$

which is compact by choice of $\epsilon$. Also,

$$
X \cdot \nu_{N}=\phi(u) \nu \cdot \nu_{N} \geq 0
$$

at all points of $\partial N$ by (8), since $\phi$ is nonnegative everywhere.

It remains only to show that $\Psi_{X} \leq-\eta|X|$. Let $q$ be any point in $N$. Let $\mathbf{e}_{1}, \ldots, \mathbf{e}_{n-1}$ be principal directions in $\operatorname{Tan}_{q} \Sigma_{q}$ corresponding to the principal curvatures $k_{1}(q), \ldots, k_{n-1}(q)$. Consider the bilinear form $Q$ on $\operatorname{Tan}_{q} N$ 
given by

$$
Q(\mathbf{u}, \mathbf{v})=\mathbf{u} \cdot \nabla_{\mathbf{v}} X
$$

We wish to calculate the matrix for $Q$ with respect to the orthonormal basis $\mathbf{e}_{1}, \ldots, \mathbf{e}_{n-1}, \nu$.

Note that if $\mathbf{v}$ is tangent to $\Sigma_{q}$, then

$$
\nabla_{\mathbf{v}} X=\nabla_{\mathbf{v}}(\phi(u) \nu)=\phi(u) \nabla_{\mathbf{v}} \nu
$$

since $\phi(u)$ is constant on $\Sigma_{q}$. Thus, if $\mathbf{u}$ and $\mathbf{v}$ are both tangent to $\Sigma_{q}$, then

$$
Q(\mathbf{u}, \mathbf{v})=\phi(u) \mathbf{u} \cdot \nabla_{\mathbf{v}} \nu=-\phi(u) B(\mathbf{u}, \mathbf{v})
$$

where $B$ is the second fundamental form of $\Sigma_{q}$ with respect to the normal $\nu$. In particular,

$$
Q\left(\mathbf{e}_{i}, \mathbf{e}_{j}\right)= \begin{cases}-\phi(u) k_{i} & \text { if } i=j \\ 0 & \text { if } i \neq j\end{cases}
$$

Since $\|\nu\| \equiv 1$, we see that $\nabla_{\mathbf{v}} \nu$ is perpendicular to $\nu$ and thus

$$
Q\left(\nu, \mathbf{e}_{i}\right)=0
$$

by (11). Since $\nu$ is the gradient of the distance function, $\nabla_{\nu} \nu=0$. Thus,

$$
\begin{aligned}
\nabla_{\nu} X & =\nabla_{\nu}(\phi(u) \nu) \\
& =\phi^{\prime}(u)\left(\nabla_{\nu} u\right) \nu+\phi(u) \nabla_{\nu} \nu \\
& =\phi^{\prime}(u) \nu
\end{aligned}
$$

so $Q(\nu, \nu)=\phi^{\prime}(u)$ and $Q\left(\mathbf{e}_{i}, \nu\right)=0$.

Hence, we see that the matrix for $Q$ with respect to the orthonormal basis $\mathbf{e}_{1}, \ldots, \mathbf{e}_{n-1}, \nu$ is a diagonal matrix with diagonal elements $-\phi(u) k_{i}$ (for $1 \leq i \leq n-1$ ) and $\phi^{\prime}(u)$. Note that

$$
-\phi(u) k_{1} \geq-\phi(u) k_{2} \geq \cdots \geq-\phi(u) k_{n-1} \geq-\phi(u) K \geq \phi^{\prime}(u)
$$

by (6), (9) and (10), since $\phi \geq 0$. In particular, since $\phi(u)=|X|$, the largest $m$ eigenvalues of $Q$ are $-|X(q)| k_{i}$ where $1 \leq i \leq m$. It follows by elementary 
linear algebra that

$$
\begin{aligned}
\Psi(q) & =\max _{P} \operatorname{trace}(Q \mid P) \\
& =-|X(q)|\left(k_{1}(q)+\cdots+k_{m}(q)\right) \\
& \leq-\eta|X(q)|
\end{aligned}
$$

by (7). This completes the proof.

Theorem 3 (Maximum Principle for Set-Theoretic Limits of Minimal Varieties). Suppose $N_{i}$ is a sequence of smooth Riemannian $n$ manifolds with boundary, and suppose that the $N_{i}$ converge smoothly to a limit Riemannian manifold $N$. Suppose for each $i$ that $V_{i}$ is an $m$ dimensional varifold in $N_{i}$ that minimizes area to first order in $N_{i}$, and suppose that the sets $\operatorname{spt}\left(V_{i}\right)$ converge to a limit set $S \subset N$. Then $S$ does not contain any point of $\partial N$ at which $N$ is strongly m-convex.

Proof. Since the result is local, we may assume that the $N_{i}$ and $N$ are all the same as smooth manifolds but have Riemannian metrics $g(i)$ and $g$ where $g(i)$ converges smoothly to $g$.

Let $\kappa_{1}(\cdot) \leq \cdots \leq \kappa_{n-1}(\cdot)$ be the principal curvatures of $\partial N$ with respect to the inward pointing unit normal.

Let $p$ be a point of $\partial N$ at which $N$ is strongly $m$-convex (with respect to $g$.) Let $0<\eta<\kappa_{1}(p)+\cdots+\kappa_{m}(p)$. In the proof of Theorem 2, we constructed a smooth function $u: N \rightarrow \mathbf{R}$ with the following properties (with respect to the metric $g$ ):

(i) $u(p)=0$ and $u>0$ on $N \backslash\{p\}$.

(ii) The set $C=\{u \leq \epsilon\}$ is compact.

(iii) $\kappa_{1}+\cdots+\kappa_{m}>\eta$ at all points of $C \cap \partial N$.

(iv) $\nabla u$ never vanishes on $C$, and

$$
k_{1}(q)+\cdots+k_{m}(q)>\eta
$$

at each point $q \in C$, where $k_{1}(q) \leq \cdots \leq k_{n-1}(q)$ are the principal curvatures of the level set $\Sigma_{q}=\{x: u(x)=u(q)\}$ with respect to the unit normal $\nabla u(q)$.

By the smooth convergence $g(i) \rightarrow g$, these properties will also hold with respect to the metric $g(i)$ for all sufficiently large $i$. Fix such an $i$. We claim that spt $V_{i}$ cannot contain any point of $C$. For if it did, the function $u$ 
restricted to $C \cap \operatorname{spt} V_{i}$ would attain a minimum at some point $q$. By (iii) and by Theorem 1, $q$ cannot be in $\partial N$. By (iv), the set $\{u \geq u(q)\}$ is strongly $m$-convex at $q$, which contradicts Theorem 1 (since $q \in \operatorname{spt} V_{i} \subset\{u \geq u(q)\}$.) Thus, $C \cap \operatorname{spt} V_{i}$ is empty. Since $p$ is in the interior of $C$, we are done.

In the case of hypersurfaces, we get a stronger result:

Theorem 4 (Strong Maximum Principle for Minimal Hypersurfaces). Suppose that $N$ is a smooth Riemannian manifold (not necessarily complete) with boundary, that $\partial N$ is connected, and that $N$ is mean convex, i.e., that

$$
H \cdot \nu_{N} \geq 0
$$

on $\partial N$, where $H$ is the mean curvature vector of $\partial N$ and where $\nu_{N}$ is the unit normal to $\partial N$ that points into $N$. Let $m=\operatorname{dim}(N)-1$, and suppose that $V$ is an $m$-dimensional varifold that minimizes area to first order in $N$.

1) If spt $V$ contains any point of $\partial N$, then it must contain all of $\partial N$ and $H$ must vanish everywhere on $\partial N$.

2) If $V$ is a stationary integral varifold, then $V$ can be written as $W+W^{\prime}$ where the support of $W$ is $\partial N$ and the support of $W^{\prime}$ is disjoint from $\partial N$.

Proof. Assertion (1) was proved by Solomon and White [7]. Assertion (1) also follows rather directly from Theorem 1: see [7, Step 1, p. 687] and the comments at the end of [7].

To prove (2), we may assume that $\partial N$ is a minimal hypersurface. (Otherwise spt $V$ is disjoint from $\partial N$ by assertion 1 , so we can let $W=0$ and $W^{\prime}=V$.) Let $d$ be the smallest integer such that there is a point $p \in \partial N$ at which the density of $V$ is $d$. Let $W$ be the $m$-dimensional integral varifold whose support is $\partial N$ and whose density is $d$ at every point of $\partial N$. Then $\mu_{W} \leq \mu_{V}$, so (since $V$ and $W$ are rectifiable varifolds) $W \leq V$ (as measures on the Grassman bundle.) Thus, the signed measure $W^{\prime}:=V-W$ is in fact a positive measure, i.e., a varifold. Since $V$ and $W$ are stationary integral varifolds, so is $W^{\prime}$. By choice of $d$, the varifold $W^{\prime}$ has density 0 at least one point $p$ of $\partial N$. It follows that $p$ is not in the support of $W^{\prime}$ (because the density is $\geq 1$ at every point in the support of a stationary integral varifold). But then by assertion (1), spt $W^{\prime}$ is disjoint from $\partial \mathrm{N}$.

Assertion (2) need not hold if $V$ is not an integer-multiplicity varifold. For example, let $N$ be a closed half space in $\mathbf{R}^{3}$, let $P_{i}(i=1,2,3, \ldots)$ be a 
sequence of planes in the interior of $N$ that converge to $\partial N$, let $V_{i}$ be the varifold corresponding to $P_{i}$ with multiplicity $2^{-i}$ and let $V$ be the sum of the $V_{i}$.

See $[3,5]$ for other strong maximum principles for varieties of codimension 1. In particular, Ilmanen [3] gives a very general strong maximum principle for pairs of codimension 1 minimal varieties, both of which may be singular.

Theorem 5 (Maximum Principle for Varieties with Bounded Mean Curvature). Let $N$ be a smooth Riemannian manifold with boundary and $h$ be a nonnegative number. Suppose $V$ is an $m$-dimensional varifold in $N$ and that

$$
\delta V(X)+h \int|X| d \mu_{V} \geq 0
$$

for every compactly supported $C^{1}$ vectorfield on $N$ such that

$$
X \cdot \nu_{N} \geq 0 \text { at all points of } \partial N \text {. }
$$

Then the support of $V$ cannot contain any point $p$ in $\partial N$ at which

$$
\kappa_{1}+\cdots+\kappa_{m}>h
$$

where $\kappa_{1} \leq \kappa_{2} \leq \cdots \leq \kappa_{n-1}$ are the principal curvatures of $\partial N$ with respect to the unit normal $\nu_{N}$ that points into $N$.

Indeed, there is an $\epsilon=\epsilon(h)$ such that $\operatorname{dist}(p, \operatorname{spt} V) \geq \epsilon$ for all $m$-varifolds $V$ satisfying (12).

In order to understand the meaning of the hypothesis on $V$, suppose that $V$ is a smooth $m$-dimenisonal submanifold. In that case, inequality (12) holds for all compactly supported $C^{1}$ vectorfields if and only if the length of the mean curvature vector of $V$ is everywhere bounded by $h$. The inequality holds for all $X$ satisfying (13) if and only if: (i) at every point in $V \backslash \partial N$, the length of the mean curvature vector is at most $h$, and (ii) at every point $q \in V \cap \partial N$, the mean curvature vector at $q$ can be written as the sum of a vector of length at most $h$ and a normal vector to $\partial N$ that points out of $N$.

Theorem 5 follows from Theorem 2 exactly as Theorem 1 did. (One chooses the $\eta$ in Theorem 2 to be strictly between $h$ and $\kappa_{1}(p)+\cdots+$ $\kappa_{m}(p)$.) Note that Theorem 1 is Theorem 5 in the special case $h=0$. 
Theorem 6 (Maximum Principle for Set-Theoretic Limits of Varieties with Bounded Mean Curvature). Suppose that $N_{i}$ is a sequence of smooth Riemannian n-manifolds with boundary, and suppose that the $N_{i}$ converge smoothly to a limit Riemannian manifold $N$. Suppose for each $i$ that $V_{i}$ is an m-dimensional varifold in $N_{i}$ and that

$$
\delta V_{i}(X)+h \int|X| d \mu_{V_{i}} \geq 0
$$

for every compactly supported $C^{1}$ vectorfield $X$ on $N_{i}$ such that

$$
X \cdot \nu_{N_{i}} \geq 0 \text { at all points of } \partial N_{i}
$$

Suppose also that the sets $\operatorname{spt}\left(V_{i}\right)$ converge to a limit set $S \subset N$. Then $S$ does not contain any point of $\partial N$ at which

$$
\kappa_{1}+\cdots+\kappa_{m}>h
$$

where $\kappa_{1} \leq \kappa_{2} \leq \cdots \leq \kappa_{n-1}$ are the principal curvatures of $\partial N$ with respect to the unit normal that points into $N$.

The proof is almost identical to the proof of Theorem 3.

Theorem 7 (Strong Maximum Principle for Hypersurfaces with Bounded Mean Curvature). Let $N$ be a smooth Riemannian manifold with boundary. Suppose that $\partial N$ is connected and that the mean curvature of $\partial N$ with respect to the inward pointing normal is everywhere $\geq h$, where $h>0$. Let $m=$ $\operatorname{dim}(N)-1$ and suppose $V$ is an $m$-dimensional varifold in $N$ and that

$$
\delta V(X)+h \int|X| d \mu_{V} \geq 0
$$

for every compactly supported $C^{1}$ vectorfield on $N$ such that

$$
X \cdot \nu_{N} \geq 0 \text { at all points of } \partial N .
$$

1) If spt $V$ contains any point of $\partial N$, then it must contain all of $\partial N$ and $\partial N$ must have constant mean curvature $h$.

2) If $V$ is an integral varifold with mean-curvature $\leq h$, then $V$ can be written as $W+W^{\prime}$ where the support of $W$ is $\partial N$ and the support of $W^{\prime}$ is disjoint from $\partial N$.

The proof is similar to the proof of Theorem 4, except that one uses Theorem 5 in place of Theorem 1. 


\section{Acknowledgment}

This research was supported by the NSF under grant DMS-0707126.

\section{References}

[1] W.K. Allard, On the first variation of a varifold, Ann. Math. 95(2) (1972), 417-491. MR0307015, Zbl 0252.49028.

[2] U. Dierkes and D. Schwab, Maximum principles for submanifolds of arbitrary codimension and bounded mean curvature, Calc. Var. Partial Differential Equations 22(2) (2005), 173-184. MR2106766.

[3] T. Ilmanen, A strong maximum principle for singular minimal hypersurfaces, Calc. Var. Partial Differential Equations, 4(5) (1996), 443-467. MR1402732.

[4] L.P. Jorge and F. Tomi, The barrier principle for minimal submanifolds of arbitrary codimension, Ann. Global Anal. Geom. 24(3) (2003) 261267. MR1996769.

[5] R. Schätzle, Quadratic tilt-excess decay and strong maximum principle for varifolds, Ann. Sc. Norm. Super. Pisa Cl. Sci. 3(5) (2004), no. 1, 171-231. MR2064971.

[6] L. Simon, Lectures on geometric measure theory, Proceedings of the Centre for Mathematical Analysis, 3, Australian National University, Australian National University Centre for Mathematical Analysis, Canberra, 1983. MR756417, Zbl 0546.49019.

[7] B. Solomon and B. White, A strong maximum principle for varifolds that are stationary with respect to even parametric elliptic functionals, Indiana Univ. Math. J. 38(3) (1989), 683-691, MR1017330, Zbl 0711.49059 .

[8] B. White, Which ambient spaces admit isoperimetric inequalities for submanifolds?, J. Differential Geom. 83(1) (2009), 213-228. MR2545035.

Department of Mathematics

STANFORD UNIVERSITY

BUILDING 380

StANFORD, CALIFORNIA 94305

E-mail address: white@math.stanford.edu

Received August 3, 2009 